\title{
Results of the Neuroblastoma-2015 Protocol for Treating Neuroblastoma in China: Experience from Multiple Centers
}

Yan Jin ( $\sim$ jinyan19900713@163.com )

Tianjin Tumor Hospital https://orcid.org/0000-0001-6032-8727

Jie Yan

Tianjin Medical University Cancer Institute and Hospital

Fu Li

Qilu Children's Hospital of Shandong University

Lian Jiang

the Fourth Hospital of Hebei Medical University

Jun Lu

Children's Hospital of Soochow University

Jianxin Li

Wuhan Children's Hospital

Yunpeng Dai

Shandong Provincial Hospital Affiliated to Shandong University

Jianghua Zhan

Tianjin Children's Hospital

\section{Yanna Cao}

Tianjin Medical University Cancer Institute and Hospital

\section{Zhongyuan Li}

Tianjin Medical University Cancer Institute and Hospital

Yun Liu

Tianjin Medical University Cancer Institute and Hospital

Daowei Wang

Tianjin Medical University Cancer Institute and Hospital

Zhanglin Li

Tianjin Medical University Cancer Institute and Hospital

Qiang Zhao

Tianjin Medical University Cancer Institute and Hospital

Jie Li

Tianjin Medical University Cancer Institute and Hospital 
Research article

Keywords: neuroblastoma, therapy, stem cell transplantation, radiation, Child, China

Posted Date: April 9th, 2020

DOI: https://doi.org/10.21203/rs.3.rs-20699/v1

License: (c) (i) This work is licensed under a Creative Commons Attribution 4.0 International License. Read Full License 


\section{Abstract}

ABSTRACT Background: The collaborative efforts of the Chinese Children's Cancer Group (CCCG) have led to advances in our understanding of NB biology, a standardized classification, and stratified strategies, resulting in improved outcomes in China. We aimed to assess the NB-2015 protocol formulated by the CCCG in 2015 and evaluate the extent of the impact of autologous peripheral blood stem cell transplantation (APBSCT) .

Methods: This study enrolled patients with NB between 2015 and 2018 from seven medical centers in China. The clinical characteristics of one hundred sixty-one patients were reviewed, and the therapeutic effect was evaluated as the impact on survival rate.

Results: The 3-year event-free survival (EFS) rates of the low-, intermediate-, and high-risk groups were $95.5 \%, 91.6 \%, 51.1 \%$, respectively. In total, 3 out of 7 (42.8\%) patients with stage $2 \mathrm{~B}$ disease, none of whom had MYCN amplifications, relapsed. A total of 47 of the 78 patients in the high-risk group underwent external-beam radiation to the primary tumor bed (dose ranging from 18 to $36 \mathrm{~Gy}$ ). The therapeutic effect was significantly different between patients who received radiation therapy and those who did not (3-year OS P =0.041). Within the high-risk group, The 3-year EFS rates of the patients with and without APBSCT were $72.6 \%$ and $37.1 \%$, respectively $(P=0.008)$.

Conclusions: This study showed that using the NB-2015 protocol achieved satisfactory results without the occurrence of fatal complications. For patients in the stage 2B group, chemotherapy should be intensified. APBSCT can effectively improve the survival rate of patients with NB, especially high-risk patients.

\section{Introduction}

Neuroblastoma (NB) is the third most common cancer in children after leukemia and brain cancer[1]. NB is a complex heterogeneous disease that arises from the embryonic cells that form the primitive neural crest, with a natural history ranging from a benign course to a terminal illness and from spontaneous regression without intervention to highly fatal chemoresistant disease. NB accounts for $7-10 \%$ of all pediatric malignancies but is responsible for nearly $15 \%$ of pediatric cancer deaths because of the aggressive nature of the disease and the high likelihood of metastatic disease at diagnosis[2-3]. More than half of pediatric NB patients present with disseminated metastases at initial diagnosis, and most of these patients have advanced stage disease with poor prognoses. Patients can be divided into low-, intermediate-, and high-risk groups based on the International Neuroblastoma Staging System (INSS) criteria, which consider biological and clinical features such as age at diagnosis; disease stage; tumor histology, including grade of neuroblastic differentiation and mitosis-karyorrhexis index (MKI); and molecular markers such as MYCN oncogene amplification. Most patients with INSS stage 1 and 2 NB (usually in the low-risk group) can be cured by surgery alone and have excellent prognoses, which is the consensus around the world[4-5]. However, the treatment for patients with stage 3 and 4 NB, especially 
those in the high-risk group, is still a challenge, and the outcomes are poor despite modern treatment strategies. Most of the studies show that the survival rate of patients with high-risk NB is approximately $50 \%$ with combination treatment that includes intensive chemotherapy, surgery, autologous stem cell transplantation, radiotherapy, immunotherapy and so on[6-9]. Although modern therapeutic treatments have improved the survival outcomes, these strategies still fail to eradicate the tumor in some patients with high-risk NB.

Racial and geographical disparities also exist in the risk status and survival of children with NB[10], and these disparities were thought to be due to genetic and economic differences. For decades, no relatively unified treatment protocol for NB existed in China; delayed diagnosis, lack of access to accurate staging, risk stratification, and optimal treatment, and abandonment led to survival rates much lower than those in high-income countries. Any of the abovementioned factors could lead to a poor survival rate. To standardize treatment and improve the survival of NB patients in China, several medical centers of the Chinese Children's Cancer Group (CCCG) have joined our treatment group and have used the NB-2015 protocol since 2015. Our NB-2015 protocol recommends combination treatment that includes chemotherapy, surgery, autologous peripheral blood stem cell transplantation (APBSCT), radiotherapy, and maintenance treatment with cis-retinoic acid based on the different INSS risk groups. The present article reports the results of the first retrospective study conducted by the various CCCG medical centers on NB treatment. This retrospective analysis of patients with NB who followed our NB-2015 protocol from 2015 to 2018 was performed to demonstrate the clinical features and treatment effects and to evaluate whether the therapeutic protocol had an impact on the survival rate.

\section{Materials And Methods}

\section{Patient population}

The NB-2015 protocol was carried out by the Pediatric Oncology Department of Tianjin Medical University Cancer Institute and Hospital starting in January 2015. This study was approved by the Institutional Review Board of Tianjin Medical University Cancer Institute and Hospital, and all guardians of the patients provided written informed consent. The records of Tianjin Medical University Cancer Institute and Hospital, Qilu Children's Hospital of Shandong University,the Fourth Hospital of Hebei Medical University,Children's hospital of Soochow university,Wuhan Children's Hospital,Tongji Medical College, Huazhong University of Science \& Technology, Shandong Provincial Hospital Affiliated to Shandong University and Tianjin Children's Hospital between January 1, 2015 and July 31, 2018 were searched. The follow-up period ended on July 31, 2018.

One hundred sixty-one patients with previously untreated NB were reviewed in our research. Tumors were diagnosed according to the INSS criteria. The histological findings were defined by the International Neuroblastoma Pathologic Classification (INPC) guidelines as described previously[11]. The Pathology Department of Tianjin Medical University Cancer Institute and Hospital was consulted regarding all patient pathological sections. The diagnosis was established by two pathologists in the department. The 
biological factors of the tumor such as the histopathologic classification, MYCN gene status and DNA index were analyzed together. Eligible patients were staged according to the INSS criteria and assigned to the low-, intermediate-, or high-risk group according to the Children's Oncology Group (COG) risk stratification scheme (Table 1). All patients were confirmed to have NB and adequate clinical data. All patients followed a treatment regimen based on the NB-2015 protocol that included neoadjuvant chemotherapy, surgery, adjuvant chemotherapy, APBSCT and radiotherapy according to the different risk groups determined by a professional team of pediatric oncologists. Information regarding patient characteristics was retrospectively reviewed (Table 2). The treatment protocol for each group is shown in Figs. 1. Local irradiation was administered to patients in the high-risk group.

Table 1

COG Neuroblastoma Risk Group Staging System

\begin{tabular}{|c|c|c|c|c|c|}
\hline INSS stage & Age & MYCN amplification & INPC & DNA index & Risk group \\
\hline 1 & $0-21 y$ & & & & Low-risk \\
\hline \multirow[t]{4}{*}{$2 \mathrm{~A} / 2 \mathrm{~B}$} & $<365 d$ & & & & Low-risk \\
\hline & $\geq 365 d-21 y$ & negtive & & & Low-risk \\
\hline & $\geq 365 d-21 y$ & positive & favorable & & Low-risk \\
\hline & $\geq 365 d-21 y$ & positive & unfavorable & & High-risk \\
\hline \multirow[t]{5}{*}{3} & $<365 d$ & negitive & & & intermediate-risk \\
\hline & $<365 d$ & positive & & & High-risk \\
\hline & $\geq 365 d-21 y$ & negitive & favorable & & intermediate-risk \\
\hline & $\geq 365 d-21 y$ & negitive & unfavorable & & High-risk \\
\hline & $\geq 365 d-21 y$ & positive & & & High-risk \\
\hline \multirow[t]{3}{*}{4} & $<548 d$ & negtive & & & intermediate-risk \\
\hline & $<365 d$ & positive & & & High-risk \\
\hline & $\geq 548 d-21 y$ & & & & High-risk \\
\hline \multirow[t]{4}{*}{$4 S$} & $<365 d$ & negitive & favorable & $>1$ & Low-risk \\
\hline & $<365 d$ & negitive & & 1 & intermediate-risk \\
\hline & $<365 d$ & negitive & unfavorable & & intermediate-risk \\
\hline & $<365 d$ & positive & & & High-risk \\
\hline
\end{tabular}


Table 2

Chemotherapy regimens for NB

\begin{tabular}{|c|c|c|c|c|}
\hline Risk group & Regimen & Drug & Dosage & Days \\
\hline \multirow[t]{9}{*}{ Low } & \multirow[t]{2}{*}{$\mathrm{CbE}$} & CBP & $560 \mathrm{mg} / \mathrm{m}^{2}(<12 \mathrm{~kg}: 18 \mathrm{mg} / \mathrm{kg})$ & d1 1 \\
\hline & & VP-16 & $120 \mathrm{mg} / \mathrm{m}^{2}(<12 \mathrm{~kg}: 4 \mathrm{mg} / \mathrm{kg})$ & d1-3 \\
\hline & \multirow[t]{3}{*}{$\mathrm{CbAC}$} & $\mathrm{CBP}$ & 560 mg/m² (<12 kg: 18 mg/kg) & d1 1 \\
\hline & & CTX & $1.0 \mathrm{~g} / \mathrm{m}^{2}(<12 \mathrm{~kg}: 33 \mathrm{mg} / \mathrm{kg})$ & d1 \\
\hline & & E-ADM & $30 \mathrm{mg} / \mathrm{m}^{2}(<12 \mathrm{~kg}: 1 \mathrm{mg} / \mathrm{kg})$ & $\mathrm{d} 1$ \\
\hline & \multirow[t]{2}{*}{ CE } & CTX & $1.0 \mathrm{~g} / \mathrm{m}^{2}(<12 \mathrm{~kg}: 33 \mathrm{mg} / \mathrm{kg})$ & $\mathrm{d} 1$ \\
\hline & & VP-16 & $120 \mathrm{mg} / \mathrm{m}^{2}(<12 \mathrm{~kg}: 4 \mathrm{mg} / \mathrm{kg})$ & d1-3 \\
\hline & \multirow[t]{2}{*}{ CA } & CTX & $1.0 \mathrm{~g} / \mathrm{m}^{2}(<12 \mathrm{~kg}: 33 \mathrm{mg} / \mathrm{kg})$ & $\mathrm{d} 1$ \\
\hline & & E-ADM & $30 \mathrm{mg} / \mathrm{m}^{2}(<12 \mathrm{~kg}: 1 \mathrm{mg} / \mathrm{kg})$ & d1 \\
\hline \multirow[t]{8}{*}{ Intermediate } & \multirow[t]{4}{*}{ OPEC } & VCR & $1.5 \mathrm{mg} / \mathrm{m}^{2}(<12 \mathrm{~kg}: 0.05 \mathrm{mg} / \mathrm{kg})$ & d1 1 \\
\hline & & DDP & $90 \mathrm{mg} / \mathrm{m}^{2}(<12 \mathrm{~kg}: 3 \mathrm{mg} / \mathrm{kg})$ & d2 \\
\hline & & VP-16 & $160 \mathrm{mg} / \mathrm{m}^{2}(<12 \mathrm{~kg}: 5.3 \mathrm{mg} / \mathrm{kg})$ & $\mathrm{d} 4$ \\
\hline & & CTX & $1.2 \mathrm{~g} / \mathrm{m}^{2}$ (< $\left.12 \mathrm{~kg}: 40 \mathrm{mg} / \mathrm{kg}\right)$ & $\mathrm{d} 1$ \\
\hline & \multirow[t]{4}{*}{ OPAC } & VCR & $1.5 \mathrm{mg} / \mathrm{m}^{2}(<12 \mathrm{~kg}: 0.05 \mathrm{mg} / \mathrm{kg})$ & $\mathrm{d} 1$ \\
\hline & & DDP & $90 \mathrm{mg} / \mathrm{m}^{2}(<12 \mathrm{~kg}: 3 \mathrm{mg} / \mathrm{kg})$ & $\mathrm{d} 2$ \\
\hline & & E-ADM & $30 \mathrm{mg} / \mathrm{m}^{2}(<12 \mathrm{~kg}: 1 \mathrm{mg} / \mathrm{kg})$ & $\mathrm{d} 4$ \\
\hline & & CTX & $1.2 \mathrm{~g} / \mathrm{m}^{2}(<12 \mathrm{~kg}: 40 \mathrm{mg} / \mathrm{kg})$ & d1 1 \\
\hline \multirow[t]{6}{*}{ High } & \multirow[t]{2}{*}{$\mathrm{Cl}$} & CTX & $400 \mathrm{mg} / \mathrm{m}^{2}(<12 \mathrm{~kg}: 13.3 \mathrm{mg} / \mathrm{kg})$ & d1-5 \\
\hline & & Irinotecan & $120 \mathrm{mg} / \mathrm{m}^{2}(<12 \mathrm{~kg}: 4 \mathrm{mg} / \mathrm{kg})$ & d1-3 \\
\hline & \multirow[t]{2}{*}{ PE } & DDP & $50 \mathrm{mg} / \mathrm{m}^{2}(<12 \mathrm{~kg}: 1.66 \mathrm{mg} / \mathrm{kg})$ & d1-4 \\
\hline & & VP-16 & $200 \mathrm{mg} / \mathrm{m}^{2}(<12 \mathrm{~kg}: 6.67 \mathrm{mg} / \mathrm{kg})$ & d1-3 \\
\hline & \multirow[t]{2}{*}{$\operatorname{COA}$} & CTX & $1800 \mathrm{mg} / \mathrm{m}^{2}(<12 \mathrm{~kg}: 60 \mathrm{mg} / \mathrm{kg})$ & d1-2 \\
\hline & & VCR & $<12$ mon: $0.017 \mathrm{mg} / \mathrm{kg}$ & d1-3 \\
\hline
\end{tabular}




\begin{tabular}{|c|c|c|c|c|}
\hline \multirow[t]{4}{*}{ Risk group } & Regimen & Drug & Dosage & Days \\
\hline & & & $>12 \mathrm{mon}$ and $>12 \mathrm{~kg}: 0.67 \mathrm{mg} / \mathrm{m}^{2}$ & \\
\hline & & & $>12$ mon and $<12 \mathrm{~kg}: 0.022 \mathrm{mg} / \mathrm{kg}$ & \\
\hline & & E-ADM & $25 \mathrm{mg} / \mathrm{m}^{2}(<12 \mathrm{~kg}: 0.83 \mathrm{mg} / \mathrm{kg})$ & d1-3 \\
\hline
\end{tabular}

\section{Treatment Response}

Disease status and response to treatment were categorized as complete remission, very good partial remission (VGPR), partial remission (PR), stable disease (SD), or progressive disease (PD) according to the published International Neuroblastoma Response Criteria [12]. Complete response (CR) was defined as no evidence of the primary tumor and no evidence of metastases on imaging. Very good partial remission (VGPR) was defined as a decrease in tumor size of more than $90 \%$. Partial response (PR) was defined volume reduction of more than $50 \%$ in the primary tumor and all measurable metastatic sites. Stable disease (SD) was defined as no new lesions with $<50 \%$ reduction but a $<25 \%$ size increase in any lesion; progressive disease (PD) was defined as a greater than $25 \%$ size increase in any pre-existing lesion or any new lesion. If surgical resection was attempted, the result was recorded according to the International Neuroblastoma Response Criteria: surgery was considered complete, near complete (leaving minimal residual disease, less than $5 \%$ ), incomplete (between $5 \%$ and $50 \%$ disease remaining), or as a biopsy only (more than $50 \%$ remaining).

\section{Statistical analysis}

SPSS 24.0 statistical software (Chicago, IL, USA) was used to analyze the data. Cumulative survival analysis was performed by the Kaplan-Meier method, and the log-rank test was used for single-factor analysis. Event-free survival (EFS) was calculated from the date of study enrollment until the date of the first occurrence of relapse, $\mathrm{PD}$, secondary malignancy, or death from any cause or until the date of last contact if no event occurred. Overall survival (OS) and the time to an event was calculated from the date of study enrollment until death from any cause or until the date of last contact with the patient. APBSCTrelated mortality was defined as death from any cause within 100 days post-transplantation. All $P$ values were two-tailed, and $P<0.05$ was considered statistically significant.

\section{Results}

\section{Patient characteristics}

One hundred sixty-one patients with NB were treated according to the NB-2015 protocol, including 79 male patients and 82 female patients ( $\mathrm{M}: \mathrm{F}=0.96: 1)$. The patient characteristics are listed in Table 3 . The median age at diagnosis was 37 months (range from 1 month to 141 months). In total, 51 (31.7\%) 
patients were younger than 18 months of age at diagnosis, and $110(68.3 \%)$ patients were older than 18 months of age at diagnosis. We confirmed that patient age at the time of NB diagnosis is an important independent prognostic factor. Among these patients, 47 (29.2\%), 36 (22.4\%), and 78 (48.4\%) belonged to the low-, intermediate-, and the high-risk groups, respectively. Thirty-eight (23.6\%) patients had INSS stage 1 disease, 5 (3.1\%) patients had stage 2A disease, 7 (4.3\%) had stage 2B disease, 32 (19.9\%) had stage 3 disease, and $78(48.4 \%)$ had stage 4 disease. Only one (0.6\%) patient had stage 4 S disease. MYCN amplification was observed in 12 analyzed tumors. Among these patients, only one patient had stage 1 disease, 3 patients had stage 3 disease, and the other 8 patients had stage 4 disease. In total, 47 (60.2\%) patients were in the high-risk group. We found that in our study, only patients with gross or microscopic residual disease after surgery received local irradiation to the tumor bed (dose ranging from 18 Gy to $36 \mathrm{~Gy})$. A total of $34(43.6 \%)$ patients in the high-risk group underwent autologous peripheral blood stem cell transplantation, including 18 patients who underwent tandem transplantation with busulfan + melphalan (MM) and CBP + VP16 + CTX (CEC). The other 16 patients underwent a single transplantation with MM. Patients in the high-risk group who were planning to undergo tandem transplantation received local irradiation after the first transplantation.(Table 3)

Table 3

Patients' characteristics $(n=161)$

\begin{tabular}{|lll|}
\hline Patients' characteristics & N(\%) \\
\hline Histologic type & Neuroblastoma & $112(69.6 \%)$ \\
\cline { 2 - 3 } Sex & Ganglioneuroblastoma & $49(30.4 \%)$ \\
& $\begin{array}{l}\text { Male } \\
\text { Female }\end{array}$ & $79(49.1 \%)$ \\
\hline Age at diagnosis & $<18$ months & $82(50.9 \%)$ \\
\hline \multirow{2}{*}{ INSS stage } & $\geq 18$ months & $12(7.5 \%)$ \\
& 1 & $139(86.3 \%)$ \\
& 2 & $38(23.6 \%)$ \\
& 3 & $12(7.4 \%)$ \\
& 4 & $32(19.9 \%)$ \\
\hline Risk groups & 4 s & $78(48.4 \%)$ \\
& Low & $1(0.6 \%)$ \\
\cline { 2 - 3 } & Intermediate & $47(29.2 \%)$ \\
\cline { 2 - 3 } & High & $36(22.4 \%)$ \\
\cline { 2 - 3 } & & $78(48.4 \%)$ \\
\hline
\end{tabular}




\section{Treatment Outcomes}

Up to the cut-off date for this analysis, no patients had been lost to follow-up. Patient age at the time of diagnosis is an important independent prognostic factor. There was a significant difference between patients $\triangle 18$ months and $\geq 18$ months of age (3-year OS $P=0.004$; 3-year EFS $P=0.000$ ). The 3-year OS rates and EFS rates of the low-, intermediate-, high-risk groups were $100 \%, 95.5 \%$, and $97.2 \%$ and $91.6 \%$, $65.6 \%$ and $51.1 \%$, respectively. Within the high-risk group, the 3-year OS rates of the patients with and without transplantation were $79.0 \%$ and $58.3 \%$, respectively $(P=0.014)$. The 3-year EFS rates of the patients with and without transplantation were $72.6 \%$ and $37.1 \%$, respectively $(P=0.008)($ Fig. 2,3$)$. The median survival time (MST) was 26 months (range 7-65 months). In the transplant group, no adverse effects specifically related to transplantation were observed up to 3 years after treatment. A total of 139 patients were still alive without recurrence, and 32 patients relapsed. Twenty-two patients died of disease progression after recurrence. The other 10 patients were still alive, including 3 with stage 2B disease, 1 with stage 3 disease, and 6 with stage 4 disease. Among these 10 patients, $5(50 \%)$ regained CR after further treatment. Although the patients experienced therapy-induced complications, nearly all patients could tolerate the intensive treatment. None of the patients died of complications related to transplantation. In total, 47 of the 78 patients in the high-risk group underwent external-beam radiation to the primary tumor bed, and a dose ranging from 18 to 36 Gy was applied. The 3-year EFS rate was not significantly different between patients who received radiation therapy and those who did not $(P=0.354)$. However, the 3-year OS rate was significantly different between the two groups $(P=0.041)$. Among the patients who underwent total resection, the 3-year OS rate was also significantly different between patients with and without radiation therapy $(P=0.044)$. (Fig. 4)

\section{Discussion}

Patient age at the time of NB diagnosis is a known important independent prognostic factor. Among the 161 patients in this study, 51 patients were $<18$ months old and the other 110 patients were $\geq 18$ months old at diagnosis. The OS and EFS rates were significantly different between the two groups (3-year OS $P$ $=0.004$; 3-year EFS $P=0.000$ ). These results are consistent with those of a previously published study[13].

Low-risk disease, including stage 1 and asymptomatic stage 2 disease, has an excellent prognosis after nonmutilating surgery alone, And surgical removal of the tumor may be the only treatment required for these patients. However, patients with intermediate-risk tumors may require chemotherapy to shrink the tumor before a complete surgical removal can be performed. Chemotherapy can halt rapid tumor progression, relieve life-threatening symptoms, and improve tumor resectability. Patients with low- and intermediate-risk NB have an estimated OS rate of over $90 \%$ with a continuous trend towards minimizing therapy[14]. In our study, the 3-year OS and EFS rates for the low-risk group were $100 \%$ and $95.5 \%$, respectively. The 3-year OS and EFS rates for the intermediate-risk group were $97.2 \%$ and $91.6 \%$, respectively. The patients classified as INSS stage 1 with completely resected tumors rarely relapsed and did not require postoperative chemotherapy, similar to the results of other studies[4-5]. 
Altogether, 32 (19.9\%) patients relapsed during the treatment or follow-up period. Most of these patients $(87.5 \%, 28 / 32)$ had stage 4 disease, and only 1 patient had stage 3 disease. In total, 3 out of the 7 $(42.8 \%)$ patients classified as INSS stage 2B relapsed. The histopathological type of the three stage 2B patients were all unfavorable, and none of the patients had an MYCN amplification. As shown in Fig. 2, the EFS of patients with stage 2B disease was much lower than that of patients with stage 3 disease, which may be due to low chemotherapy intensity for patients in this group or the different genotypes associated with poor prognosis, resulting in poor therapeutic effects. Molecular genetic techniques should be used to stratify patients and to optimize treatment.

Local radiotherapy has been documented to achieve local control in patients with stage 4 or high-risk stage 3 NB through induction chemotherapy and tandem stem cell transplants[15]. According to our protocol, all patients belonging to the high-risk group are required to receive radiotherapy to the primary site and persistent metastatic foci. In our retrospective analysis, we found that only 47 of the 78 patients in the high-risk group underwent external-beam radiation to the primary tumor bed, where a dose ranging from 18 to $36 \mathrm{~Gy}$ was applied. On the one hand, the reason for the poor compliance with our treatment may be that radiotherapy is more challenging to administer for children than for adults, considering factors such as poor coordination and the need for sedation. On the other hand, this difference can be partially attributed to the lack of professional pediatric radiologists in some medical centers. There were no significant differences in the 3-year EFS rates between patients who received radiation therapy and those who did not $(P=0.354)$. However, the 3-year OS was significantly different between these two patient groups $(P=0.041)$. Particularly for patients who underwent total resection, the 3-year OS rate was significantly different between patients who received radiation and those who did not $(P=0.044)$. Although patients with gross or microscopic residual disease after surgery did not receive local radiation to the tumor bed, high-intensity chemotherapy played a role. Our study showed that for high-risk patients with NB, even after complete excision, local radiation was also necessary.

The incorporation of three distinct phases of therapy has improved outcomes for high-risk NB: intensive induction treatment, myeloablative chemotherapy with autologous hematopoietic stem cell rescue, and treatment of minimal residual disease (MRD). The survival outcomes of high-risk NB patients have continually improved in recent years, which is attributed to the intensification of therapeutic approaches[16-18]. Even after such therapies, the relapse rate remains higher than $40 \%$, which has led to MRD-positive patients using post-transplant treatment such as isotretinoin and monoclonal anti-GD2 antibodies[19]. China has a lack of monoclonal anti-GD2 antibodies; thus, in our NB-2015 protocol, the treatment strategy for high-risk NB incorporated no more than eight cycles of induction chemotherapy, surgery, consolidation with tandem APBSCT, and post-consolidation treatment with only 13-cis-retinoic acid for 6 months. Induction therapy played a critical role in managing patients with high-risk NB and typically consisted of intensive multiagent chemotherapy and surgical resection of the primary tumor. Over the past decades, consolidation therapy with stem cell rescue for patients with initial responses to chemotherapy has become the standard of care for high-risk NB in the United States and Europe, largely based upon results from cooperative group randomized trials comparing the outcomes between this approach and an approach with chemotherapy only. Through the use of tandem autologous SCT, the 
outcome of high-risk NB patients has improved. Many different combinations of drugs for hematopoietic stem cell transplantation exist[20-22]. One clinical trial for NB patients showed that autologous hematopoietic stem cell transplantation was performed using carboplatin/etoposide/cyclophosphamide (CEC) followed by thiotepa/cyclophosphamide. Other studies have shown alternative consolidation transplantation regimens, including melphalan, MM and carboplatin/etoposide/melphalan (CEM). A recent and compelling report from the European Neuroblastoma Study Group (ENSG) suggested that MM was associated with greater EFS than CEM in a randomized comparison of patients who achieved a good response with an induction regimen[23]. Therefore, in our therapeutic protocol, we recommended tandem APBSCT with MM and CEC and observed very good effects. In our study, among the 34 patients who underwent APBSCT, 18 patients successfully received tandem transplantation. The other 16 patients failed to receive a second APBSCT after undergoing the first procedure, which was due to nonmedical reasons (parent preference, insurance limitations). In addition, we found that the tandem transplantation was well tolerated with no unbearable complications related to APBSCT, even including hepatic venoocclusive disease (HVOD) or sinusoidal obstructive syndrome (SOS). Toxicities were as expected, and the time to engraftment was rapid for both cycles. Most importantly, APBSCT obviously improved the survival of patients in the high-risk group. The 3-year OS rate of the transplantation group was $79.0 \%$, and the 3-year EFS rate of the transplantation group was $72.6 \%$. The survival outcomes were not significantly different between patients who received tandem transplantation and those who received single transplantation, which may be attributed to the small sample size and short follow-up period.

\section{Conclusion}

This study showed that the NB-2015 protocol obtained satisfactory results without the occurrence of fatal complications. Patients in the stage 2B group need further genotype testing, and the chemotherapy regimen should be intensified according to the different genotypes. Multidisciplinary treatment was essential, especially for high-risk patients, and APBSCT can effectively improve the survival rate. A longer follow-up period is needed to survey the survival rate and incidence of complications for Chinese NB patients who receive chemotherapy, radiotherapy and APBSCT. The outcomes for patients with NB have improved; however, the field continues to expand with efforts in developing more targeted therapies for high-risk patients.

\section{Declarations}

Competing interests

The authors have no conflicts of interest.

Funding

Supported by the National Key R\&D Program of China(2018YFC1313000, 2018YFC1313001).

Authors' contributions

Collection of clinical case data and follow-up of patients: Jie Yan, Jie Li, Fu Li, Lian Jiang, Jun Lu, Jianxin Li, Ping Zhao, Jianghua Zhan, Yan Jin,Yanna Cao, Zhongyuan Li,Daowei Wang and Zhanglin Li. Data 
curation: Jie Yan and Yan Jin. Statistical analysis: Yan Jin. Funding acquisition: Qiang Zhao. Writingoriginal draft: Jie Yan. Writing-review and editing: Jie Li, Qiang Zhao and Yun Liu.

Acknowledgements

Not applicable.

\section{Acknowledgements}

Not applicable.

\section{Ethics approval and consent to participate}

Written informed consent and assent forms to participate in the study were obtained from all participating subjects including children, their parents or legal guardians before enrolling in this study. This clinical study was approved by Medical Clinical Committee of Tianjin Cancer Hospital (reference number: bc2018002) according to the ethical principles of the Declaration of Helsinki (1975) including its revision.

\section{Consent for publication}

Not applicable.

\section{Availability of data and materials}

All data generated or analysed during this study are included in this published article.

\section{References}

1. Park JR, Eggert A, Caron H. Neuroblastoma: biology, prognosis, and treatment. Hematol Oncol Clin North Am. 2010;24(1):65-86.

2. Mueller S, Matthay KK. Neuroblastoma: biology and staging. Curr Oncol Rep. 2009;11(6):431-8.

3. Ward E, DeSantis C, Robbins A, Kohler B, Jemal A. Childhood and adolescent cancer statistics, 2014. CA Cancer J Clin. 2014;64(2):83-103.

4. De Bernardi B, Mosseri V, Rubie H, Castel V, et al. Treatment of localised resectable neuroblastoma. Results of the LNESG1 study by the SIOP Europe Neuroblastoma Group. $\mathrm{Br} \mathrm{J}$ Cancer. 2008;99(7):1027-33.

5. Strother DR, London WB, Schmidt ML, et al. Outcome after surgery alone or with restricted use of chemotherapy for patients with low-risk neuroblastoma: results of Children's Oncology Group study P9641. J Clin Oncol. 2012;30(15):1842-8.

6. Matthay KK, Reynolds CP, Seeger RC, et al. Long-term results for children with high-risk neuroblastoma treated on a randomized trial of myeloablative therapy followed by 13-cis retinoic acid: a children's oncology group study. J Clin Oncol. 2009;27(7):1007-13. 
7. Valteau-Couanet D, Le Deley MC, Bergeron C, et al. Long-term results of the combination of the N7 induction chemotherapy and the busulfan-melphalan high dose chemotherapy. Pediatr Blood Cancer. 2014;61(6):977-81.

8. Ladenstein R, Potschger U, Pearson ADJ, et al. Busulfan and melphalan versus carboplatin, etoposide, and melphalan as high dose chemotherapy for high-risk neuroblastoma (HRNBL1/SIOPEN): an international, randomised, multi-arm, open-label, phase 3 trial. Lancet Oncol. 2017;18(4):500-14.

9. Pearson AD, Pinkerton CR, Lewis IJ, et al. High-dose rapid and standard ACCEPTED MANUSCRIPT induction chemotherapy for patients aged over 1 year with stage 4 neuroblastoma: a randomised trial. Lancet Oncol. 2008;9(3):247-56.

10. London WB, Castleberry RP, Matthay KK, et al. Evidence for an age cutoff greater than 365 days for neuroblastoma risk group stratification in the Children's Oncology Group. J Clin Oncol. 2005;23(27):6459-65.

11. Shimada H, Ambros IM, Dehner LP, et al. The international neuroblastoma pathology classification (the Shimada system). Cancer. 1999;86(2):364-72.

12. Brodeur GM, Pritchard J, Berthold F, et al. Revisions of the international criteria for neuroblastoma diagnosis, staging, and response to treatment. J Clin Oncol. 1993;11(8):1466-77.

13. Moroz V, Machin D, Faldum A, et al. Changes over three decades in outcome and the prognostic influence of age-at-diagnosis in young patients with neuroblastoma: a report from the International Neuroblastoma Risk Group Project. Eur J Cancer. 2011;47:561-71.

14. Ahmed G, Fawzy M, Elmenawi S, et al. Role of surgery in localized initially unresectable neuroblastoma. J Pediatr Urol. 2018;14:231-6. doi:.

15. Marcus KJ, Shamberger R, Litman $\mathrm{H}$, et al. Primary tumor control in patients with stage $3 / 4$ unfavorable neuroblastoma treated with tandemdouble autologous stem cell transplants. J Pediatr Hematol Oncol. 2003 Dec;25(12):934-40.

16. Whittle SB, Smith V, Doherty E, Zhao S, McCarty S, Zage PE. Overview and recent advances in the treatment of neuroblastoma. Expert Rev Anticancer Ther. 2017;17(4):369-86.

17. Esposito MR, Aveic S, Seydel A, Tonini GP. Neuroblastoma treatment in the post genomic era. J Biomed Sci. 2017;24(1):14.

18. biology, prognosis, and treatment

Neuroblastoma

Park JR, Eggert A, Caron H. Neuroblastoma: biology, prognosis, and treatment.

19. Hematol Oncol Clin North Am 24(1) (2010) 65-86.

20. Yu AL, Gilman AL, Ozkaynak MF, et al. Anti-GD2 antibody with GM-CSF, interleukin-2, and isotretinoin for neuroblastoma. N Engl J Med. 2010;363:1324-34.

21. Pritchard J, Cotterill BA, Germond SM, Imeson J, de Kraker J, Jones DR. High dose melphalan in the treatment of advances neuroblastoma: Results of a randomized trial (ENSG-1) by the European Neuroblastoma Study Group. Ped. Blood Cancer. 2005;44(4):348-57. 
22. Ladenstein RL, Poetschger $U$, Luksch R, et al. Busulphan-melphalan as a myeloablative therapy (MAT) for high-risk neuroblastoma: Results from the HR-NBL1/SIOPEN trial. 2011 ASCO Annual Meeting. Abstract 2. Presented. Jun 5.2011.

23. Kreissman SG, Villablanca JG, Seeger RC, et al. A randomized phase III trial of myeloablative autologous peripheral blood stem cell (PBSC) transplant (ASCT) for high-risk neuroblastoma (HRNB) employing immunomagnetic purged (P) versus unpurged (UP) PBSC: A Children's Oncology Group study. J Clin Oncol (Meeting Abstracts). 2008; 26(15)(suppl):10011.

24. Ladenstein RL, Poetschger U, Luksch R, et al. Busulphan-melphalan as a myeloablative therapy (MAT) for high-risk neuroblastoma: Results from the HR-NBL1/SIOPEN trial. 2011 ASCO Annual Meeting. Abstract 2. Presented. Jun 5.2011.

\section{Figures}




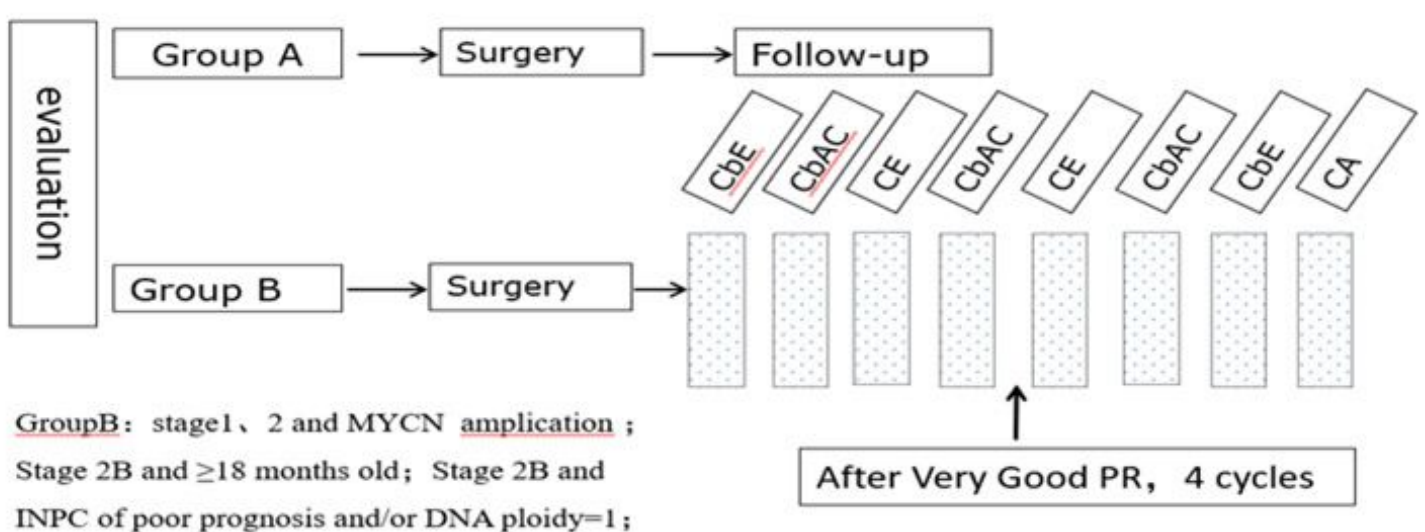

INPC of poor prognosis and/or DNA ploidy $=1$;

Stage $4 \mathrm{~s}$ with clinical symptoms

Group A : stage 1,2 except groupB

(a)
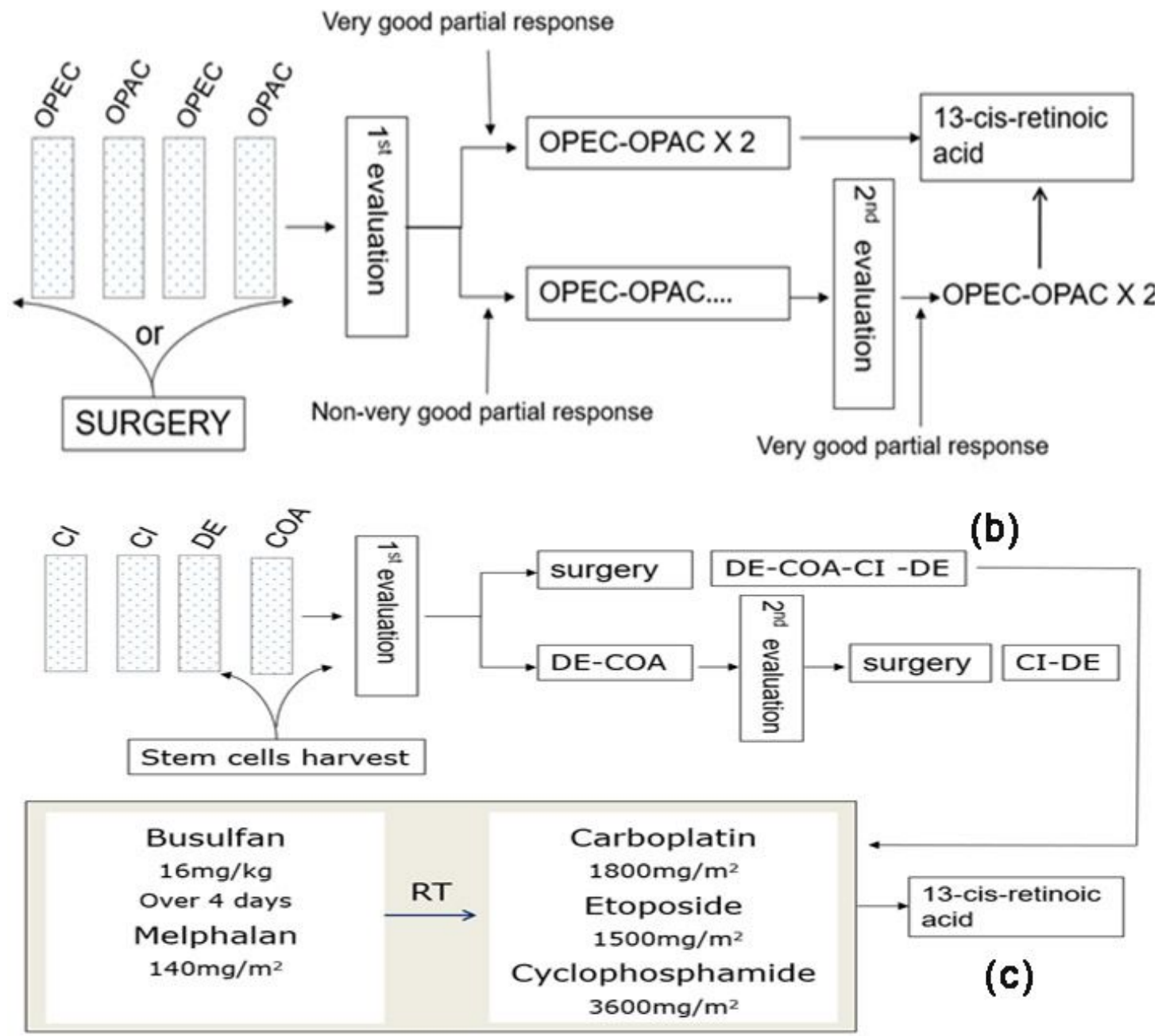

\section{Figure 1}

Figure 1 
(a)

Event Free Survival (EFS)

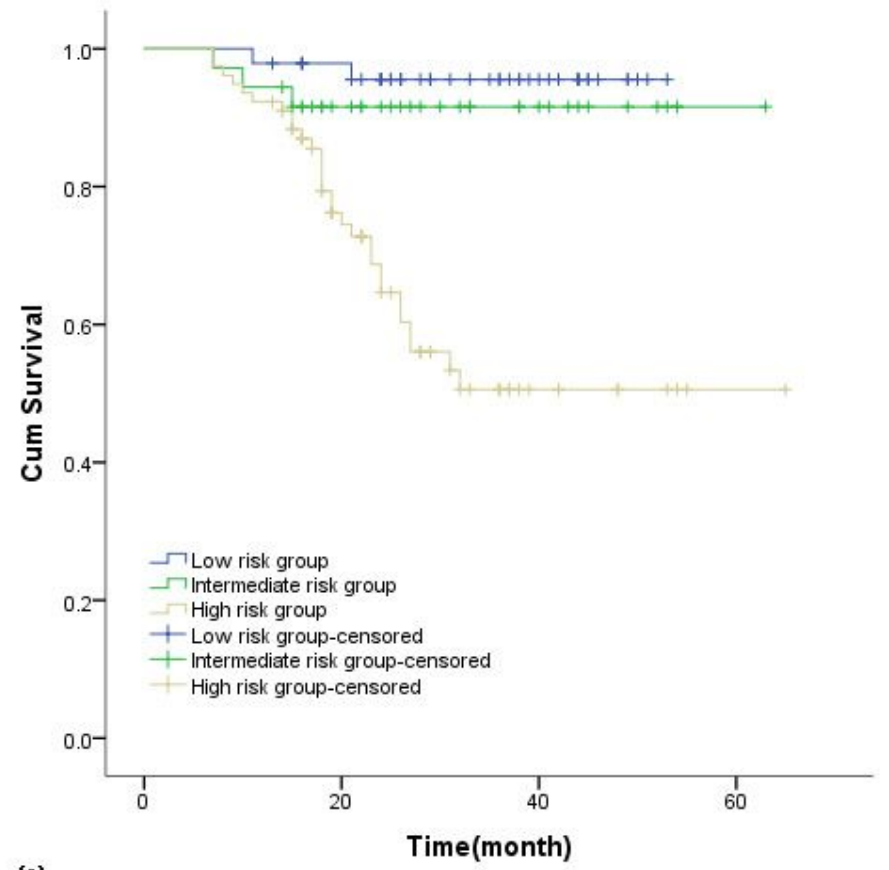

(c)

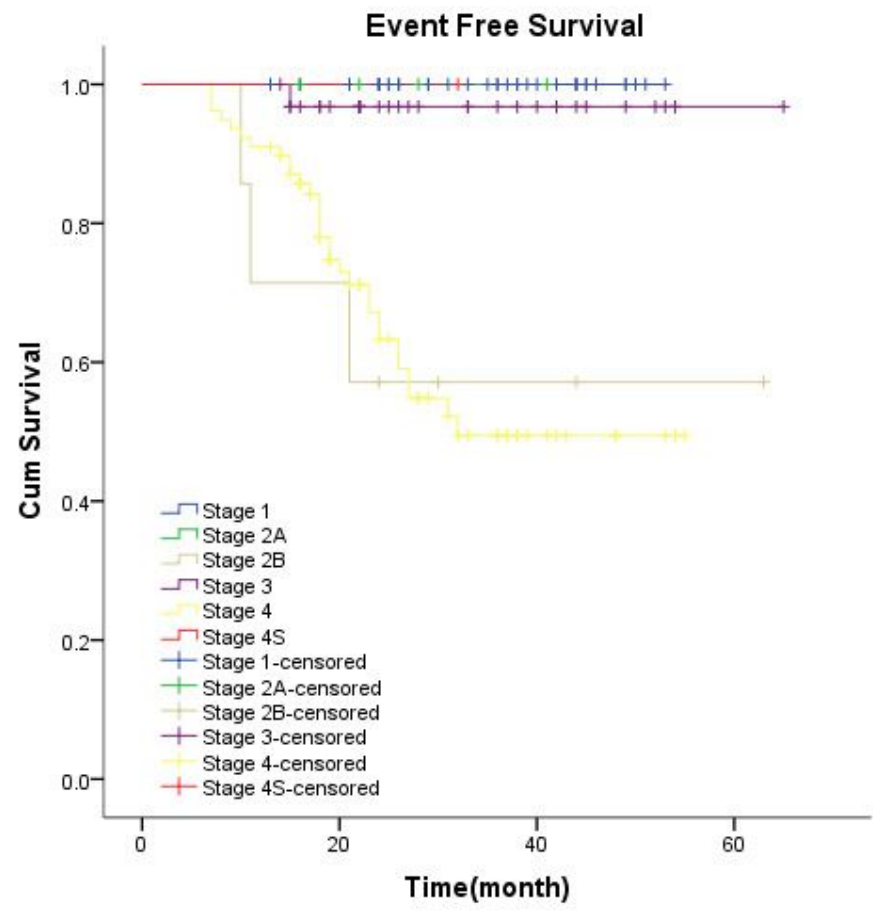

(b)

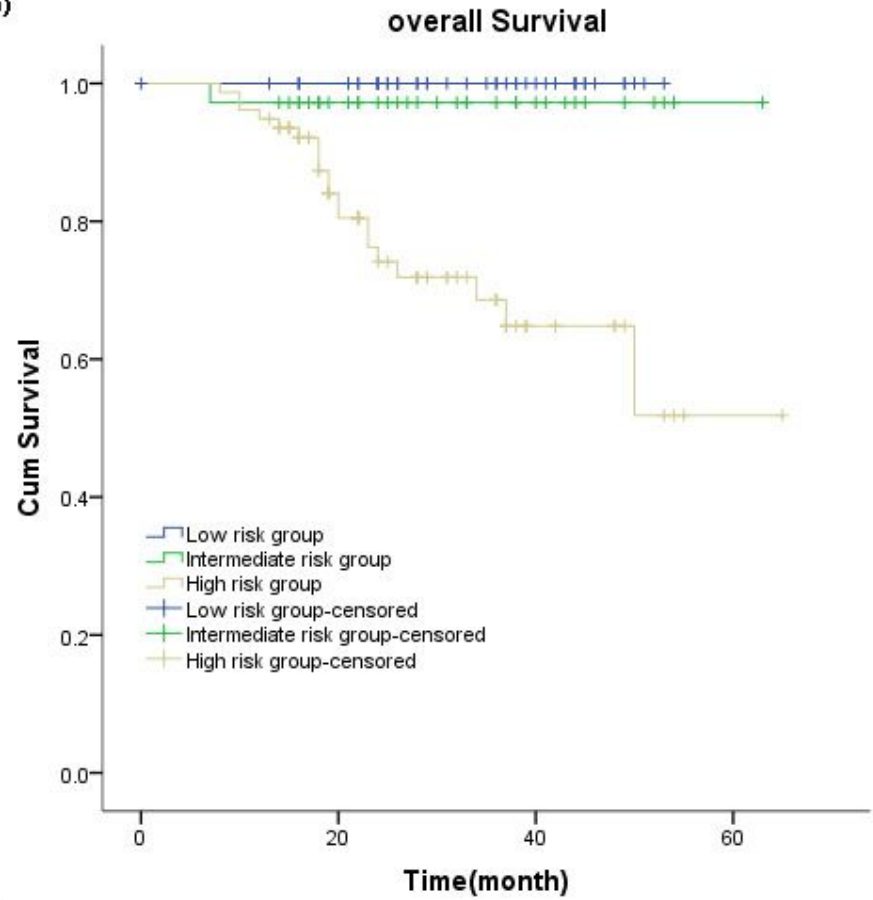

(d)

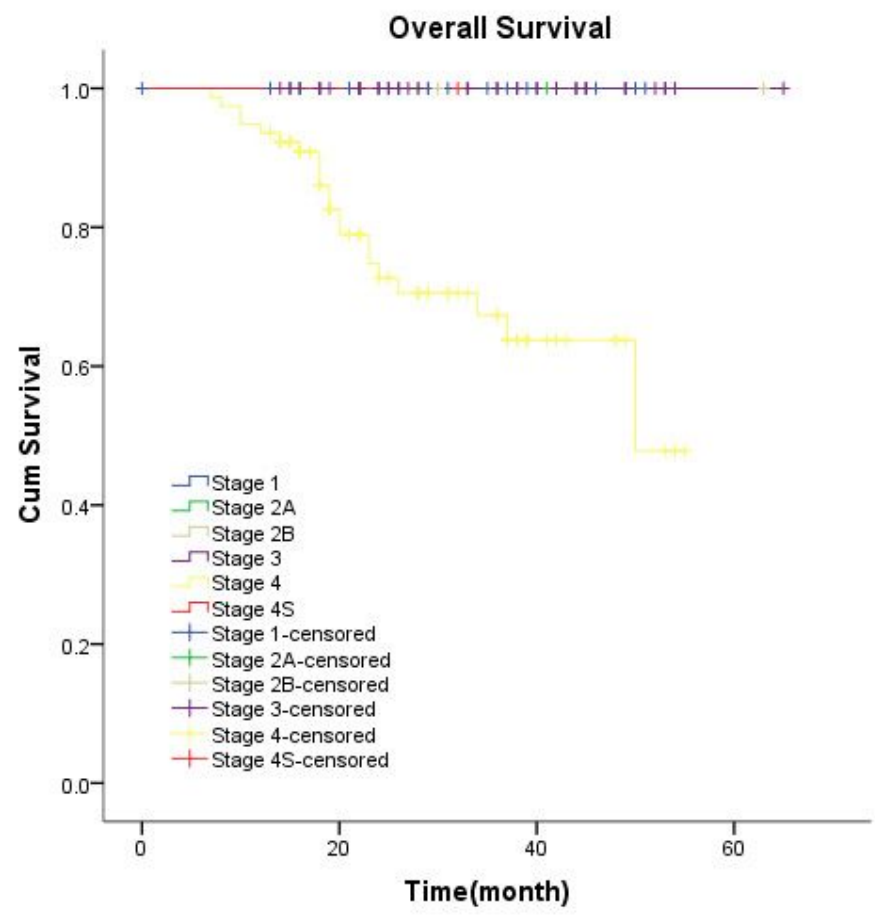

Figure 2

Figure 2 
(a)

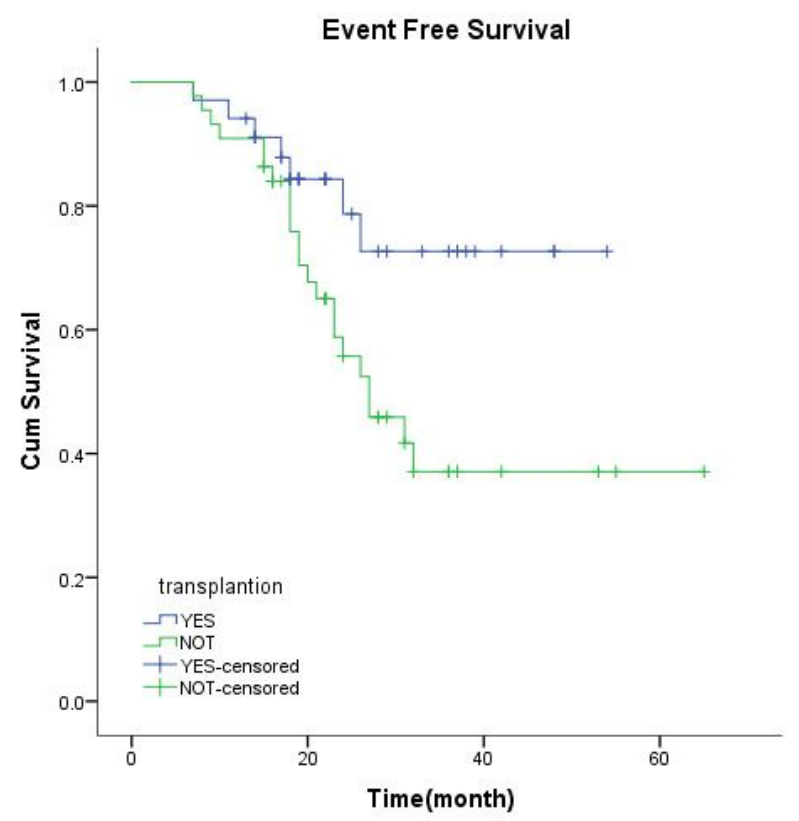

(b)

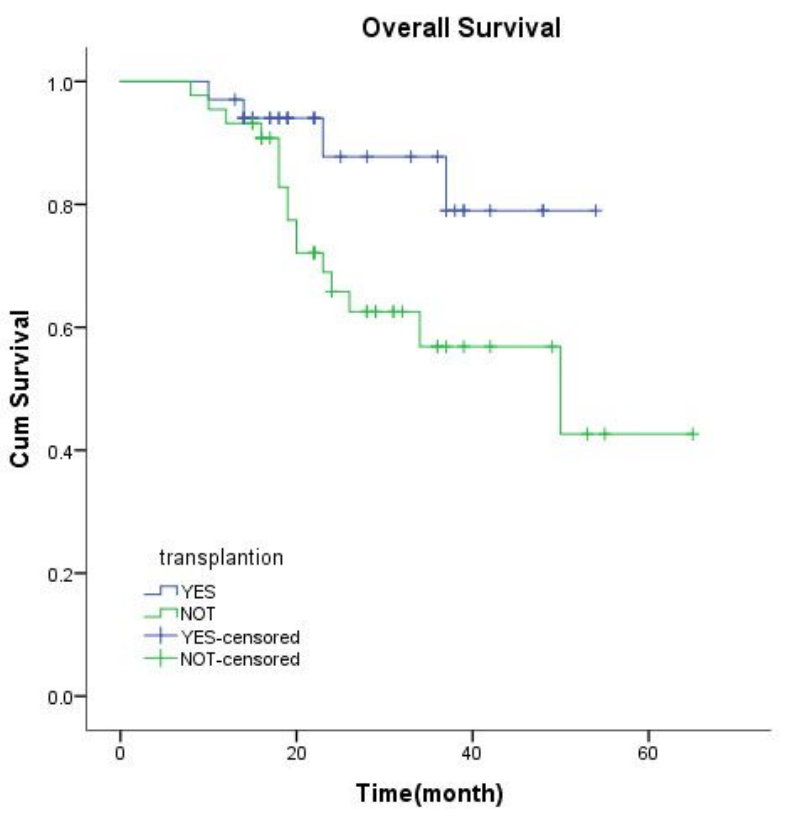

\section{Figure 3}

Figure 3 


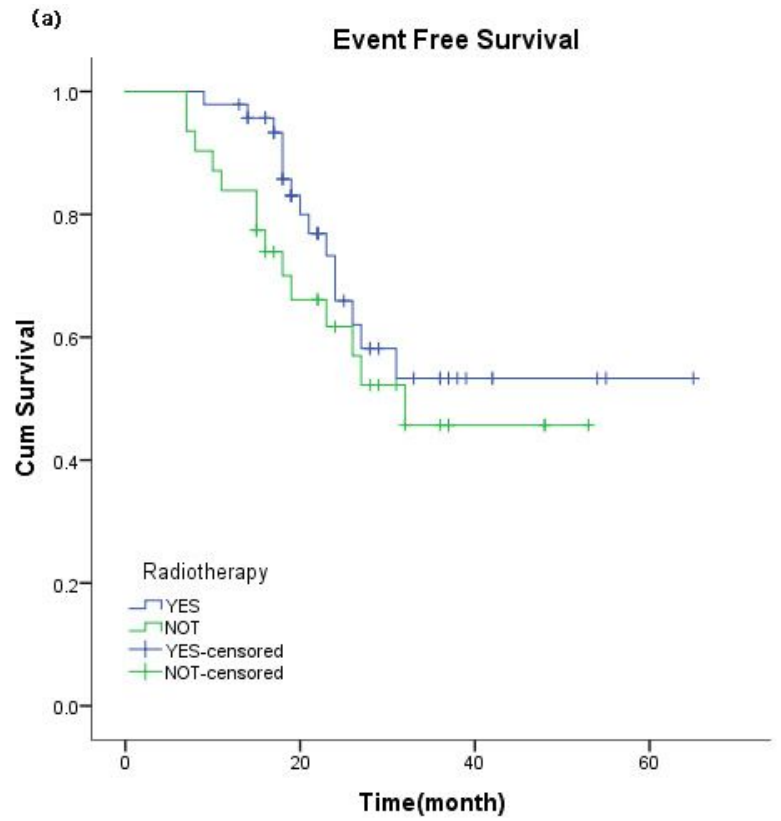

(b)

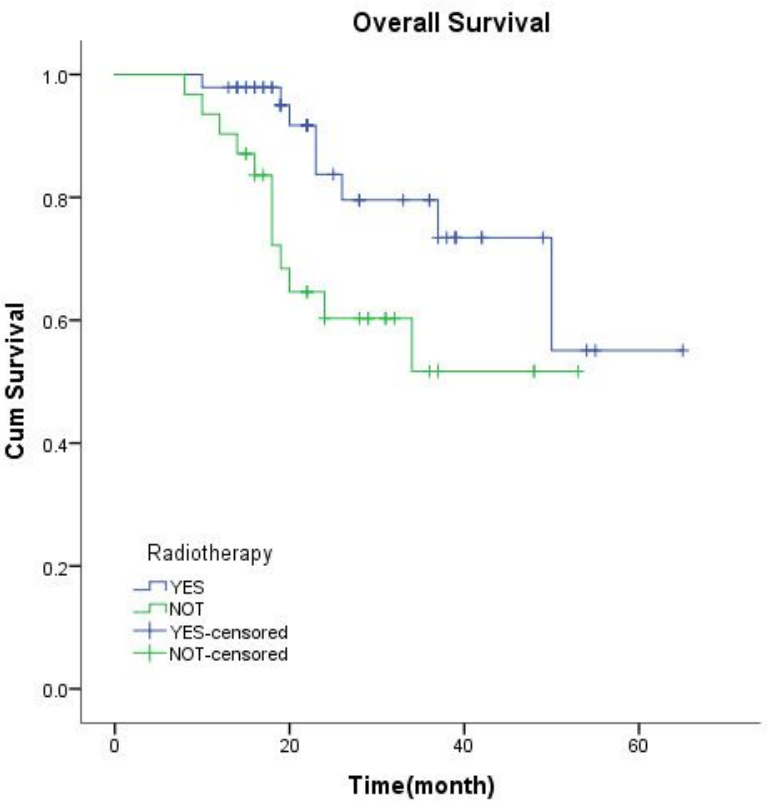

(c)

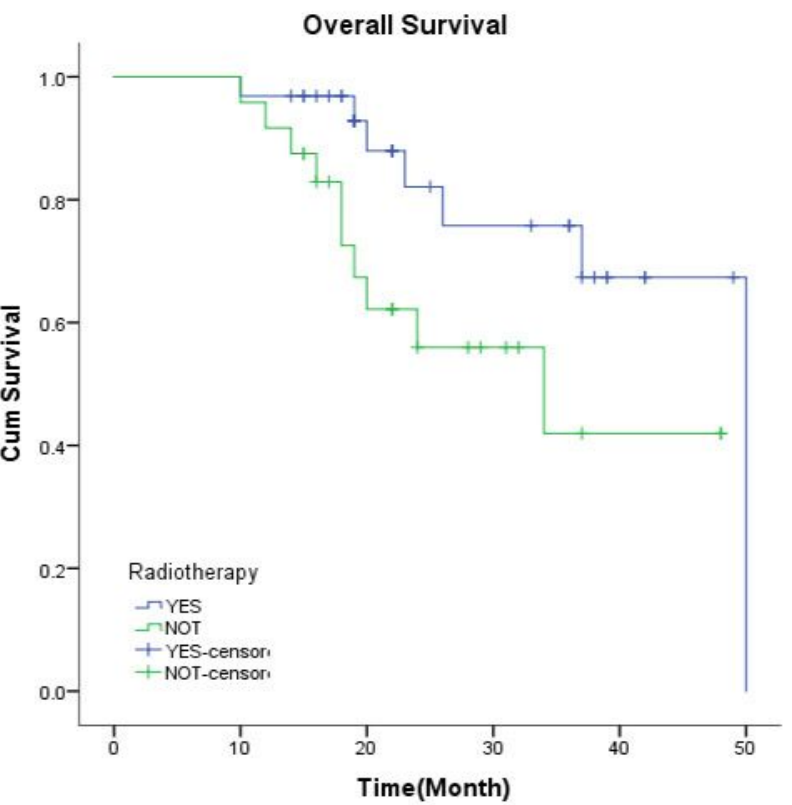

Figure 4

Figure 4

\section{Supplementary Files}

This is a list of supplementary files associated with this preprint. Click to download.

- Ethicsapprovalandconsenttoparticipate.pdf 
- EditingCertificate.pdf

Page 19/19 